\title{
A remark on the Piatetski-Shapiro-Vinogradov theorem
}

\author{
by \\ YingChun CAI (Shanghai)
}

1. Introduction. In 1937 Vinogradov [7] proved the well known Goldbach-Vinogradov theorem: Every sufficiently large odd integer $N$ can be represented as a sum of three primes. It can be stated in a more exact quantitative form: Let $T(N)$ denote the number of solutions of the equation

$$
N=p_{1}+p_{2}+p_{3} .
$$

Then

$$
T(N)=\frac{C(N) N^{2}}{2 \log ^{3} N}+O\left(\frac{N^{2}}{\log ^{A} N}\right)
$$

for any $A>3$, where $C(N)$ denotes the singular series

$$
C(N)=\prod_{p \mid N}\left(1-\frac{1}{(p-1)^{2}}\right) \prod_{(p, N)=1}\left(1+\frac{1}{(p-1)^{3}}\right) .
$$

Motivated by earlier work of Erdős and Nathanson [2] on sums of squares, some mathematicians considered the question of whether one could find thin subsets of primes which were still sufficient to obtain the GoldbachVinogradov theorem. In 1986, based on probability considerations, Wirsing [8] proved that there exists a subset $S$ of primes with the property

$$
\sum_{\substack{p \leq x \\ p \in S}} 1 \ll(x \log x)^{1 / 3},
$$

which serves this purpose. Although Wirsing's result is best possible apart from the logarithmic factor, it does not lead to a subset of primes which is constructive or recognizable.

2000 Mathematics Subject Classification: Primary 11N36.

Project supported by The National Natural Science Fundation of China (grants no. 10171060, 19801021) and by MCSEC. 
Primes of the form $\left[n^{c}\right]$, where $1 \leq c<2$, are called Piatetski-Shapiro primes. Let $\gamma=1 / c$ and

$$
P_{\gamma}=\left\{p \mid p=\left[n^{1 / \gamma}\right]=\left[n^{c}\right]\right\} .
$$

In 1992 Balog and Friedlander [1] obtained a theorem which had two interesting corollaries:

Corollary 1. For any fixed $20 / 21<\gamma \leq 1$, the Goldbach-Vinogradov theorem holds with primes in $P_{\gamma}$.

COROLlary 2. For any fixed $8 / 9<\gamma \leq 1$, the Goldbach-Vinogradov theorem holds with one prime in $P_{\gamma}$.

In 1995 Jia [4] improved Corollary 1 to

Theorem 1. For any fixed $15 / 16<\gamma \leq 1$, the Goldbach-Vinogradov theorem holds with primes in $P_{\gamma}$.

The purpose of this note is to present an approach different from that of Balog-Friedlander's which leads to an improvement of Corollary 2.

Theorem 2. For any fixed 205/243 $<\gamma \leq 1$, the Goldbach-Vinogradov theorem holds with one prime in $P_{\gamma}$.

2. Proof of Theorem 2. In order to prove Theorem 2 we need the following two lemmas.

Lemma 1 [6]. For any fixed 205/243< $<\leq 1$,

$$
P_{\gamma}(x)=\sum_{\substack{x<p \leq 2 x \\ p=\left[n^{1 / \gamma}\right]}} 1 \gg \frac{x^{\gamma}}{\gamma \log x} .
$$

Lemma 2 [3]. Let $E(x)$ denote the number of even integers in the interval $[x / 2, x]$ which cannot be represented as a sum of two primes. Then for any $A>0$,

$$
E(x)=O_{A}\left(\frac{x}{\log ^{A} x}\right) .
$$

In 1975 Montgomery and Vaughan [5] improved Lemma 2 by showing that there exists an effective constant $\Delta>0$ such that

$$
E(x)=O\left(x^{1-\Delta}\right),
$$

but for our purpose Lemma 2 is sufficient.

Proof of Theorem 2. Let

$$
\mathcal{A}=\left\{N-p \mid N / 3<p \leq 2 N / 3, p \in P_{\gamma}\right\} .
$$


Then by Lemma 1 we have

$$
|\mathcal{A}| \gg \frac{N^{\gamma}}{\gamma \log N}
$$

Let $E(\mathcal{A})$ denote the number of even integers in $\mathcal{A}$ which cannot be represented as a sum of two primes. Then by Lemma 2 we have

$$
E(\mathcal{A}) \ll \frac{N^{\gamma}}{\log ^{3} N},
$$

and

$$
|\mathcal{A} \backslash E(\mathcal{A})| \gg \frac{N^{\gamma}}{\gamma \log N} .
$$

For any $N-p \in \mathcal{A} \backslash E(\mathcal{A})$, there exist primes $p_{1}, p_{2}$ such that

$$
N-p=p_{1}+p_{2}
$$

hence

$$
N=p+p_{1}+p_{2}, \quad N / 3<p \leq 2 N / 3, p \in P_{\gamma},
$$

and Theorem 2 follows.

\section{References}

[1] A. Balog and J. Friedlander, A hybrid theorem on primes, Pacific J. Math. 156 (1992), 45-62.

[2] P. Erdös and M. B. Nathanson, Lagrange's theorem and thin subsequences of squares, in: Contributions to Probability, J. Gani and V. K. Rohatgi (eds.), Academic Press, New York, 1981, 3-9.

[3] L. K. Hua, Some results in the additive prime number theory, Quart. J. Math. Oxford 9 (1938), 68-80.

[4] C. H. Jia, On the Piatetski-Shapiro-Vinogradov Theorem, Acta Arith. 73 (1995), $1-28$.

[5] H. L. Montgomery and R. C. Vaughan, The exceptional set in Goldbach's problem, ibid. 27 (1975), 353-370.

[6] J. Rivat and J. Wu, Prime numbers of the form $\left[n^{c}\right]$, Glasgow Math. J. 43 (2001), 237-254.

[7] I. M. Vinogradov, Representation of an odd number as the sum of three primes, Dokl. Akad. Nauk SSSR 15 (1937), 291-294.

[8] E. Wirsing, Thin subbases, Analysis 6 (1986), 285-308.

Institute of Mathematics

Tongji University

Shanghai 200092, P.R. China

E-mail: yccai@mail.shu.edu.cn

Received on 26.8.2002

and in revised form on 14.11.2002 